\title{
Validation of Thwaites' Diagnostic Score in Adults with Tuberculous Meningitis
}

\author{
Dr. Hari Kishan Boorugu ${ }^{1 *}$, Dr. Varun Reddy ${ }^{2}$, Dr. Arshad M Punjani ${ }^{3}$
}

${ }^{1}$ MD General Medicine Consultant Physician, Yashoda Hospitals, Somajiguda, Hyderabad -82, Telangana, India

${ }^{2}$ MD DM Neurology Consultant Neurophysician Yashoda hospitals, Somajiguda, Hyderabad -82, Telangana, India

${ }^{3}$ MD General Medicine Consultant Physician Yashoda hospitals, Somajiguda, Hyderabad -82, Telangana, India

DOI: $10.36347 /$ sasjm.2020.v06i08.001

| Received: 04.08.2020 | Accepted: 12.08.2020 | Published: 14.08.2020

*Corresponding author: Dr. Hari Kishan Boorugu

Abstract

Original Research Article

Objective: To validate Thwaites' diagnostic score for diagnosis of tuberculous meningitis. Materials and methods: It is a retrospective study done at a tertiary teaching hospital in South India. Clinical features including CSF parameters were collected and diagnosis of tuberculous meningitis or bacterial meningitis was made by Thwaites' score and compared against final diagnosis made by a composite criteria. This is a simple score comprising of age, duration of illness, presence of leukocytosis, CSF white cell count and CSF neutrophil percentage. Results: Thwaites' score had sensitivity and specificity of $96 \%$ and $56 \%$ to diagnose tuberculous meningitis. Conclusion: Thwaites' diagnostic score is simple and helpful in detecting patients with tuberculous meningitis in population with high prevalence, especially in resource poor settings where nucleic acid amplification tests, neuroimaging are not easily available.

Keywords: Thwaites' score, tuberculous meningitis, adults.

Copyright @ 2020: This is an open-access article distributed under the terms of the Creative Commons Attribution license which permits unrestricted use, distribution, and reproduction in any medium for non-commercial use (NonCommercial, or CC-BY-NC) provided the original author and source are credited.

\section{INTRODUCTION}

Tuberculous meningitis (TBM) is a dreaded form of tuberculosis with significant morbidity and mortality. Over the years, there has been some progress in development of rapid diagnostic tools for diagnosis of pulmonary and extra pulmonary tuberculosis but we do not have an ideal diagnostic tool to diagnose TBM yet which is highly accurate, quick, inexpensive and widely available especially in resource poor settings where the disease is more prevalent. We still rely largely on clinical presentation and cerebrospinal fluid (CSF) findings to diagnose TBM and delay in diagnosis and initiation of treatment is associated with poor outcome [1].

Thwaites et al. proposed a simple clinical scoring system comprising of clinical features and CSF findings to differentiate TBM from bacterial meningitis in the year 2002 [1]. This scoring system is simple and is of reasonable accuracy and is of use especially in resource poor settings where nucleic acid amplification testing (NAAT) may not be available. NAAT has its own limitations too. We did this study to validate this scoring system in Indian population.

\section{METHODOLOGY}

This was a retrospective study done in a tertiary referral teaching hospital in south India. All the patients with the clinical and CSF features suggestive of meningitis admitted to adult medical wards between March 2003 and April 2005 were included in this study. Their clinical status and CSF acid fast bacilli (AFB) cultures were followed up at 8 weeks. Data was collected from medical records [2]. Institutional review board protocol for retrospective studies was followed.

All patients with meningitis (as suggested by CSF picture i.e., 10 cells or more per mm [3] with CSF sugar less than $50 \%$ of concomitant blood sugar) admitted to adult medical wards were enrolled in the study.

\section{Patients were excluded if}

1. if they received treatment for both pyogenic (for a minimum of 5 days) and tuberculous meningitis

2. Patients with CSF sugar $>50 \%$ of concomitant blood sugar were excluded

3. HIV patients with fungal meningitis 
Patients with culture proven meningitis or with corroborative evidence i.e. evidence of tuberculosis outside central nervous system were taken into study irrespective of the treatment received and the outcome.

Table-1: Diagnostic score (proposed by Thwaites et al)
\begin{tabular}{|l|l|}
\hline Criteria & Score \\
\hline 1. Age & \\
\hline$>=36$ & 2 \\
\hline$<36$ & 0 \\
\hline 2. Blood white cell count $\left(10^{3} / \mathrm{ml}\right)$ & \\
\hline$>=15000$ & 4 \\
\hline$<15000$ & 0 \\
\hline 3. History of illness (days) & \\
\hline$>=6$ & -5 \\
\hline$<6$ & 0 \\
\hline 4. CSF white blood cell count $\left(10^{3} / \mathrm{ml}\right)$ & \\
\hline$>=900$ & 3 \\
\hline$<900$ & 0 \\
\hline 5. CSF neutrophil percentage & \\
\hline$>=75$ & 4 \\
\hline$<75$ & 0 \\
\hline
\end{tabular}

According to Thwaites' diagnostic score, if the patient has a score of 4 or less, he or she has tuberculous meningitis, and if the patient has a score of more than 4 , he or she has bacterial meningitis.

We compared this diagnostic score against the final diagnosis of tuberculous or pyogenic meningitis. The diagnosis of TBM was made if mycobacterium tuberculosis was isolated from CSF (smear or culture positivity) or if the computerized tomography scan (CT) of brain showed features suggestive of tubercular meningitis (hydrocephalus, basal exudates), chest radiography suggestive of active pulmonary tuberculosis (PTB) or other evidence of TB outside central nervous system (eg: sputum positive for AFB, other tissues like lymph nodes positive for AFB or with granulomatous inflammation suggestive of tuberculosis on histopathology). A good response to antitubercular therapy (ATT) in the form of symptomatic improvement of headache, fever, altered sensorium at the end of 2 months was also considered diagnostic of TBM. Post meningitic sequelae were not considered as lack of treatment response.

Bacterial meningitis was diagnosed if the pathogenic bacteria were isolated from CSF (smear or culture positivity) or with clinical meningitis with all of the following features:

- Low concentration of glucose in CSF $(<50 \%$ of that in blood)

- Neutrophils in CSF above 75\%

- Recovery (without anti tuberculosis chemotherapy) at 4 weeks after admission

Sensitivity, Specificity, negative and positive predictive values, likelihood ratios was calculated using a simple 2 X 2 table.
There were 281 patients with meningitis admitted to the medical wards between March 2003 and April 2005(excluding HIV patients with fungal meningitis). Out of this, 131 patients were enrolled in the study.

\section{RESULTS}

150 patients were excluded for the following reasons

1. 34 patients - as they received treatment for both pyogenic meningitis and tuberculous meningitis (antibiotics + ATT)

2. 19 patients - as complete data was not available (this included patients for whom only ventricular CSF results were available)

3. 13 patients with aseptic meningitis

4. 9 patients who were discharged against medical advice or died.

5. 62 patients - due to lack of follow up.

6. 13 patients - as their CSF/blood sugar $>50 \%$ (though they were diagnosed to have either pyogenic or TBM by the treating physician).

Of the 131 patients enrolled, 97 patients were diagnosed to have tuberculous meningitis, 27 patients were diagnosed to have pyogenic meningitis as per the gold standard criteria mentioned above (table 4). Seven patients had meningitis of other than TB or pyogenic etiology. Out of these, 3 patients had fungal meningitis.

Thwaites' score had sensitivity of $96 \%$ and specificity of $56 \%$ in our study with predictive value for positive and negative tests around $86 \%$ (table 4) 
Table-2: Criteria supporting diagnosis of TBM

\begin{tabular}{|l|l|}
\hline Criterion & Number of cases \\
\hline CT brain characteristics TBM & 28 \\
\hline Coexisting Pulmonary tuberculosis & 16 \\
\hline Extra pulmonary tuberculosis besides CNS involvement & 18 \\
\hline CSF Culture showing AFB growth & 21 \\
\hline Diagnosis based on treatment response & 34 \\
\hline
\end{tabular}

Table-3: Patient characteristics in TBM and bacterial meningitis groups

\begin{tabular}{|l|l|l|l|l|l|}
\hline & \multicolumn{2}{|l|}{ TBM(n=97) } & \multicolumn{2}{l|}{ Bacterial Meningitis(n=27) } & p value \\
\hline & Mean & Median & Mean & Median & \\
\hline Age in years & 33.6 & 31 & 47.9 & 52 & 0.001 \\
\hline Blood WBC count & 10438 & 9700 & 17507 & 15650 & $<0.001$ \\
\hline Duration of illness in days & 55 & 30 & 4.4 & 4 & 0.001 \\
\hline CSF WBC count $\left(10^{3} / \mathrm{ml}\right)$ & 384 & 220 & 3599 & 980 & 0.009 \\
\hline CSF neutrophil \% & 19 & 7 & 73.9 & 91 & $<0.001$ \\
\hline Duration of fever in Days & 41.9 & 20 & 4 & 3 & $<0.001$ \\
\hline Duration of headache in days & 35.8 & 15 & 3.7 & 3.5 & $<0.001$ \\
\hline Serum sodium (meq/L) & 129.8 & 132 & 136.3 & 136.5 & 0.001 \\
\hline CSF sugar(mg/dL) & 34 & 33.5 & 36.2 & 25 & 0.74 \\
\hline CSF protein(mg/dL) & 275 & 169.5 & 416 & 370 & 0.13 \\
\hline CSF sugar/blood sugar ratio & 26.9 & 26.9 & 22.8 & 20 & 0.25 \\
\hline
\end{tabular}

Table-4: Comparison of diagnosis by Thwaites' score versus final diagnosis

\begin{tabular}{|l|l|l|l|l|}
\hline \multicolumn{5}{|c|}{ Diagnosis by Thwaites algorithm Vs Final diagnosis } \\
\cline { 3 - 5 } \multicolumn{2}{|c|}{} & Gold standard diagnosis & Total \\
\cline { 3 - 5 } & TBM & Nontuberculous meningitis & \\
\hline \multirow{2}{*}{$\begin{array}{l}\text { Thwaite's } \\
\text { diagnostic index }\end{array}$} & Positive & 94 & 15 & 109 \\
\cline { 2 - 5 } & Negative & 3 & 19 & 22 \\
\hline Total & 97 & 27 & 131 \\
\hline
\end{tabular}

Sensitivity $=96.9 \%$

Specificity $=56 \%$

Predictive value for a positive test $=86.23 \%$

Predictive value for a negative test $=86.36 \%$
Likelihood ratio for positive test (diagnosis of TBM) $=$ 2.197

Likelihood ratio for negative test (diagnosis of nontuberculous meningitis) $=0.055$

Table-5: CSF culture positivity in TB and bacterial meningitis

\begin{tabular}{|c|c|c|c|c|}
\hline SF culture & TBM & & Bacterial meningitis & \\
\hline & $\mathrm{N}$ & $\%$ & $\mathrm{~N}$ & $\%$ \\
\hline Positive & 21 & 21.6 & 14 & 51.8 \\
\hline Negative & 76 & 78.4 & 13 & 48.2 \\
\hline Total & 97 & 100 & 27 & 100 \\
\hline
\end{tabular}

\section{DISCUSSION}

Though this study was done in between 2003 and 2005, we felt this study is still relevant in present times as there is no easy way to diagnose TBM even today especially in areas where NAAT tests and neuro imaging is not easily available. Criteria used to make the final diagnosis (against which. Thwaites' diagnostic algorithm was compared) was similar to the criteria. Thwaites used for his original study with a few modifications [1]. Lancet consensus scoring system (for uniform diagnosis of TBM for research studies) was published in 2010 [2]. Interestingly our criteria used to make final diagnosis of TBM were similar to that of Lancet consensus scoring system (involving clinical, $\mathrm{CSF}$ and radiological criteria).

Though culture positivity is the gold standard for diagnosis of TBM, culture yield is less than $50 \%$ in most studies [3]. In our study, culture yield for TBM was only $21 \%$ and $35 \%$ in Thwaites' study (table 4 ). About $28 \%$ of patients had neuroimaging criteria to support diagnosis of TBM, one third of TBM patients had evidence of pulmonary or extra pulmonary tuberculosis (besides CNS involvement) and response to anti tuberculosis treatment (ATT) was used to diagnose TBM in one third of patients (table 1). As expected, significantly higher proportion of people in bacterial meningitis group had peripheral leucocytosis and CSF neutrophil predominance (table 2). Thwaites' score had sensitivity of $96 \%$ and specificity of $56 \%$ in our study with predictive value for positive and negative tests around $86 \%$ (table 3). Mustafa Sunbul et al. evaluated Thwaites' score and sensitivity and specificity to diagnose TBM were $95 \%$ and $70 \%$ respectively [4]. In our study if only TBM and pyogenic meningitis were included for analysis (excluding 
cryptococcal meningitis etc), sensitivity and specificity of Thwaites' score to diagnose TBM were $96 \%$ and $70 \%$ respectively which was very similar to the findings of Mustafa Sunbul et al. Zhang et al. reported a sensitivity and specificity of $98.2 \%$ and $43.6 \%$ respectively [5]. In a study published by Roshin Kurien et al., Thwaites' score compared well with Lancet consensus score in diagnosing TBM.[6] Duration of illness was given the maximum weightage in Thwaites' score to differentiate TBM from bacterial meningitis. Mean duration of illness in TBM group in Thwaites' study and our study were 12 days and 55 days respectively (table 2). Much longer duration of illness at presentation probably indicates delayed health seeking behaviour in our population. Another interesting finding in our study was significant difference in incidence of hyponatremia between TBM and pyogenic meningitis (43\% and 13\%). Tarek Dendale et al. used serum sodium as one of the differentiating features between TBM and bacterial meningitis in their diagnostic algorithm [7]. In a study done by Singh et al., hyponatremia was found in $65 \%$ of patients with TBM and the biochemical features were suggestive of SIADH [8]. NAAT are now available which are useful in diagnosis of TBM. In a review article published by Garg RK et al., sensitivity of Xpert MTB/RIF ranged between $19-59 \%$ in various studies [3]. In a study done by Fiona $\mathrm{V}$ Creswell to assess the accuracy of various tests for TBM in HIV patients, sensitivity of Xpert MTB/RIF Ultra, Xpert MTB/RIF and MGIT culture were $76 \%, 55 \%$ and $61 \%$ respectively[9]. Negative predictive value of Xpert MTB/RIF Ultra test was $93 \%$ and hence cannot be used as a rule out test. Limitations of Xpert MTB tests are cost, availability and moderate sensitivity. However, results can be obtained quickly and can help confirming the diagnosis and also provide information on Mycobacterial susceptibility to Rifampicin. Considering limitations of various modalities, as of now, composite criteria seem to be most practical and useful method to diagnose TBM. NAAT tests combined with clinical criteria like Thwaites' score and neuroimaging can be very useful in quick diagnosis of TBM and prompt initiation of ATT which is important in reducing morbidity and mortality. Thwaites' score has very good sensitivity and most patients with TBM can be diagnosed promptly using this score. A few patients may receive unnecessary anti tuberculous treatment if this score alone is used for diagnosis which may be acceptable in resource limited setting where TBM is prevalent, as we know delay in treatment is associated with significant increase in morbidity and mortality.

\section{CONCLUSION}

Lancet consensus scoring system is the most widely accepted algorithm to diagnose TBM. NAAT and neuroimaging findings which are integral part of Lancet consensus score may not be easily available in many settings where prevalence of TB is high. Treatment cannot be delayed till the culture results are available. In resource poor countries with high prevalence of TB, Thwaites' score appears to be useful in diagnosing TBM though a few patients without TBM may receive ATT if initiated using this criteria.

\section{REFERENCES}

1. Thwaites GE, Chau TT, Stepniewska K, Phu NH, Chuong LV, Sinh DX, White NJ, Parry CM, Farrar JJ. Diagnosis of adult tuberculous meningitis by use of clinical and laboratory features. The Lancet. 2002 Oct 26;360(9342):1287-92.

2. Marais S, Thwaites G, Schoeman JF, Török ME, Misra UK, Prasad K, Donald PR, Wilkinson RJ, Marais BJ. Tuberculous meningitis: a uniform case definition for use in clinical research. The Lancet infectious diseases. 2010 Nov 1;10(11):803-12.

3. Garg RK. Microbiological diagnosis of tuberculous meningitis: Phenotype to genotype. The Indian Journal of Medical Research. 2019 Nov; $150(5): 448$

4. Sunbul M, Atilla A, Esen S, Eroglu C, Leblebicioglu H. Thwaites' diagnostic scoring and the prediction of tuberculous meningitis. Medical Principles and Practice. 2005;14(3):151-4.

5. Zhang YL, Lin S, Shao LY, Zhang WH, Weng XH. Validation of Thwaites' diagnostic scoring system for the differential diagnosis of tuberculous meningitis and bacterial meningitis. Japanese Journal of Infectious Diseases. 2014;67(6):428-31.

6. Kurien R, Sudarsanam TD, Samantha S, Thomas K. Tuberculous meningitis: a comparison of scoring systems for diagnosis. Oman medical journal. 2013 May;28(3):163.

7. Kurien R, Sudarsanam TD, Samantha S, Thomas K. Tuberculous meningitis: a comparison of scoring systems for diagnosis. Oman medical journal. 2013 May;28(3):163.

8. Dendane T, Madani N, Zekraoui A, Belayachi J, Abidi K, Zeggwagh AA, Abouqal R. A simple diagnostic aid for tuberculous meningitis in adults in Morocco by use of clinical and laboratory features. International Journal of Infectious Diseases. 2013 Jun 1;17(6):e461-5.

9. Singh BS, Patwari AK, Deb M. Serum sodium and osmolal changes in tuberculous meningitis. Indian Pediatr. 1994;31(11):1345- 1350.

10. Cresswell FV, Tugume L, Bahr NC, Kwizera R, Bangdiwala AS, Musubire AK, Rutakingirwa M, Kagimu E, Nuwagira E, Mpoza E, Rhein J. Xpert MTB/RIF Ultra for the diagnosis of HIVassociated tuberculous meningitis: a prospective validation study. The Lancet Infectious Diseases. 2020 Mar 1;20(3):308-17. 